\title{
An evaluation of awareness and practices regarding management of avulsed teeth among Paediatricians in a district of north India
}

\author{
Dr Rajat Ahuja, Dr. Ravindra Ahuja, Dr Gagan Thakur \\ 'Junior Resident, Department of Pediatrics, Era's Lucknow Medical College and Hospital, Sarfarazganj, \\ Lucknow \\ ${ }^{2}$ Associate Professor, Department of Pediatrics, Hind Institute of Medical Sciences, Barabanki, UP. \\ ${ }^{3}$ Reader,Department of Oral and Maxillofacial Surgery, Peoples College of Dental Sciences and Research \\ Centre, Bhopal
}

\begin{abstract}
:
Objective: To evaluate the awareness and practices regarding management of avulsed teeth among paediatricians in a district of north India.

Methods: A multiple choice questionnaire comprising of 10 items was formulated to assess the knowledge, attitude and practices of medical professionals regarding first aid and management of "tooth loss" (Avulsion). The study involved a sample of 314 medical doctors who were either attached to the institutes or into private practice in the district of Lucknow, UP, India. Questionnaires were electronically mailed to 400 participants followed by a reminder email after fifteen days. If did not respond after 30 days, they were excluded from the study. The results are presented in percentages.

Results: About one third (34.7\%) of the participants had experienced children visiting to them with tooth avulsion. A large number of $62.7 \%$ participants stated that it would be wise to seek an immediate consultation with the dentist on the contrary to a small $15 \%$ who believed consultation was not required. Out of those participants who had witnessed patients reporting in their practice with avulsed tooth, $48.6 \%$ of them referred such patients to dentists immediately without intervening however, a small number of participants $(17.4 \%)$ opted to place the tooth back into the socket and refer patients to dentists.
\end{abstract}

Conclusion: A small percentage of practising about the avulsion teeth by the practitioners in this study indicates a serious problem for dental health. There is a need an educational campaign regarding this.

Key words: Avulsed teeth, Awareness, Paediatricians

\section{Introduction}

Children may experience injuries ranging from simple enamel fracture to complicated maxillofacial trauma. Epidemiological data clearly demonstrate that maxillary central and lateral incisors are the most frequently avulsed primary teeth with an incidence of between $7-12 \%{ }^{1-4}$ Consequently, paediatricians and also dentists who treat a significant number of children under 4 years of age are likely to encounter a child with an avulsed maxillary incisor and also the first to actually provide primary treatment. In order to provide adequate treatment for dental trauma the medical professionals need to know the basics of managing such conditions. Not many studies have been carried out to assess the awareness and knowledge of medical professional in assessment and providing primary care to children with trauma resulting in avulsion.

The present study was conducted to evaluate the awareness and practices regarding management of avulsed teeth among paediatricians in a district of north India.

\section{Materials And Methods}

A multiple choice questionnaire comprising of 10 items was formulated to assess the knowledge, attitude and practices of medical professionals regarding first aid and management of "tooth loss" (Avulsion). The study involved a sample of 314 medical doctors who were either attached to the institutes or into private practice in the district of Lucknow, UP, India. Questionnaires were electronically mailed to 400 participants followed by a reminder email after fifteen days. If did not respond after 30 days, they were excluded from the study. The results are presented in percentages.

\section{Results}

A total of 314 physicians agreed to participate and replied the completely filled questionnaires. About one third (34.7\%) of the participants had experienced children visiting to them with tooth avulsion. A large number of $62.7 \%$ participants stated that it would be wise to seek an immediate consultation with the dentist on the contrary to a small $15 \%$ who believed consultation was not required. However, others would either wait for 
30 minutes $(7.6 \%)$, wait for few hours $(1.3 \%)$ or until the next day $(3.5 \%)$. About one tenth $(9.9 \%)$ stated they would either prescribe analgesics and antibiotics or control bleeding and take care of lacerations, if any (Table1).

Out of those participants who had witnessed patients reporting in their practice with avulsed tooth, $48.6 \%$ of them referred such patients to dentists immediately without intervening however, a small number of participants (17.4\%) opted to place the tooth back into the socket and refer patients to dentists. However, 19.3\% provided first aid to patients but discarded the tooth at the same time and the rest $14.7 \%$ would administer tetanus toxoid to traumatised children invariably (Table-2).

\section{Discussion}

Tooth avulsion constitutes an emergency for children and adolescents and therefore necessitates management approaches ensuring survival of the avulsed tooth/teeth. Re-implantation of the avulsed tooth may be a promising treatment modality to increase the life of the tooth and reduce the economics of treatment manifold. Since physicians and paediatricians are the primary care providers for children in many situations, it is imperative for them to have knowledge about first hand management of dental trauma. In the present study, a substantial (34.7\%) of the participants had witnessed patients coming to them with the chief complaint of "tooth loss" or "fallen off" tooth yet somehow it never occurred to most of them to either seek required dental advice or attend educative programmes for the same. What could be largely appreciated was that almost all realized the importance of conserving time in such cases and referring patient for prompt evaluation by dentist in this study. According to Heithersay, the value of immediate replacement and treatment of the tooth should be a priority. Time is a critical factor in successful re-plantation. The avulsed tooth should be washed and replaced immediately. But, few $17.4 \%$ of the participants in this study were confident and readily practised placing the tooth back into the socket. Previous studies by Krishnaraj ${ }^{6}$ and Holan and Shmueli ${ }^{7}$ had reported that only $5.5 \%$ and $4 \%$ respectively have tried re-implantation.

McCann et al. ${ }^{8}$ found that physicians and medical undergraduates in the United Kingdom were inadequately educated about oral lesions, indicating serious deficiencies in diagnostic awareness. The existing health education system should provide more courses on dental and dentofacial trauma management for physicians and medical residents. Although the dentist will provide a definitive treatment to follow, it becomes essential that the medical professionals gather and posses basic knowledge of the primary management of tooth avulsion.

\section{Conclusion}

A small percentage of practising about the avulsion teeth by the practitioners in this study indicates a serious problem for dental health. There is a need an educational campaign regarding this.

\section{Acknowledgement}

The authors are thankful to those who participated in the study.

\section{Conflict of interest}

None

Source of funding

None

\section{References}

[1]. Judd PL. Paediatric dental trauma: a hospital survey. Ont Dent 1985; 62(6):19-20,23.

[2]. Borum MK, Andreasen JO. Sequelae of trauma to primary maxillary incisors. I. Complications in the primary dentition. Endod Dent Traumatol 1998; 14(1):31-44.

[3]. Onetto JE, Flores MT, Garbarino ML. Dental trauma in children and adolescents in Valparaiso, Chile. Endod Dent Traumatol 1994; 10(5):223-7.

[4]. Kinoshita S, Mitomi T, Taguchi Y, Noda T. Prognosis of replanted primary incisors after injuries. Endod Dent Traumatol 2000; $16(4): 175-83$.

[5]. Heithersay GS. Replantation of avulsed teeth. A review. Aust Dent J. 1975 Apr;20(2):63-72.

[6]. Krishnaraj Subhashraj. Awareness of management of dental trauma among medical professionals in Pondicherry, India. Dental Traumatology 2009; 25: 92-94

[7]. Holan G, Shmueli Y. Knowledge of physicians in hospital emergency rooms in Israel on their role in cases of avulsion of permanent incisors. Int J Paed Dent 2003;13:13-9.

[8]. McCann PJ, Sweeney MP, Gibson J, Bagg J. Training in oral disease, diagnosis and treatment for medical students and doctors in the United Kingdom. Br J Oral Maxfac Surg 2005;43:61-5. 
Table-1: Attitude of participants towards the patients with avulsion teeth

\begin{tabular}{|l|c|c|}
\hline \multicolumn{1}{|c|}{ Attitude* } & No. & \% \\
\hline Experienced in visiting children to them with avulsion tooth & 109 & 34.7 \\
\hline Wised to seek an immediate consultation with the dentist & 197 & 62.7 \\
\hline Consultation not required. & 47 & 15.0 \\
\hline wait for 30 mins & 24 & 7.6 \\
\hline Wait for few hours & 4 & 1.3 \\
\hline Wait for next day & 11 & 3.5 \\
\hline Prescribed analgesics and antibiotics & 31 & 9.9 \\
\hline
\end{tabular}

*Multiple response

Table-2: Practice of participants towards the patients with avulsion teeth among experienced participants

\begin{tabular}{|l|c|c|}
\hline \multicolumn{1}{|c|}{ Practice } & No. $(\mathbf{n}=\mathbf{1 0 9})$ & $\mathbf{\%}$ \\
\hline Referred child immediately & 53 & 48.6 \\
\hline Placed the tooth back into the socket and refer patients to dentists. & 19 & 17.4 \\
\hline Provided first aid to patients but discarded the tooth at the same time & 21 & 19.3 \\
\hline Administer tetanus toxoid to traumatised children invariably & 16 & 14.7 \\
\hline
\end{tabular}

